\title{
T-cell biomarkers for diagnosis of tuberculosis: candidate evaluation by a simple whole blood assay for clinical translation
}

\author{
To the Editor:
}

Diagnosis of pulmonary tuberculosis (TB) disease, the most common cause of death due to infection globally [1], depends on direct detection of Mycobacterium tuberculosis, typically from a sputum specimen. However, up to half of individuals with microbiologically proven TB do not have a prolonged productive cough [2], required to produce sputum. To address this challenge, investigators have identified candidate blood-based host biomarkers for TB diagnosis, based on $M$. tuberculosis-specific T-cell memory phenotypes, activation or cytokine expression profiles [3-9]. Such biomarkers have also shown potential as surrogates to monitor TB treatment response [3, 10]. Here, we aimed to: 1) compare the diagnostic performance of several blood-based T-cell biomarkers in TB patients and controls; 2) determine if combinations of these biomarkers improve diagnostic performance; 3) identify the minimal number of flow cytometry parameters required to distinguish active TB patients from persons with latent $M$. tuberculosis infection (LTBI); and 4) translate measurement of these biomarkers from peripheral blood mononuclear cells to whole blood to enable a simplified application in field studies.

We enrolled 25 healthy adults with LTBI, defined by QuantiFERON-TB Gold In-Tube assay (QFT; Qiagen, Hilden, Germany), and 25 HIV-negative adults with TB disease (XpertMTB/RIF+) (Cepheid, Sunnyvale, CA, USA) from a region endemic for TB in South Africa. Blood was collected prior to treatment initiation in persons with active TB and 12-18 months later, after they were declared cured $(n=19)$. In controls, blood was also collected at $12-18$ months after enrolment $(n=20)$. Whole blood was stimulated with whole mycobacteria (BCG, Connaught), ESAT-6/CFP-10 peptides from QFT tubes, and TB122 peptide pool [11] using a standardised protocol [12]. Cells were stained with the following antibodies: anti-CD3 (Beckman Coulter, Krefeld, Germany; clone UCHT1; ECD), anti-CD4 (Becton Dickinson (BD), Franklin Lakes, NJ, USA; clone RPA-T4; BV605), anti-CD8 (Biolegend, San Diego, CA, USA; clone RPA-T8; BV510), anti-IFN $\gamma$ (BD; clone B27; Alexa Fluor 700), anti-TNF (eBioScience, San Diego, CA, USA; clone Mab11; PE-Cy7), anti-IL2 (BD; clone 5344.111; FITC), anti-CD27 (BD; clone M-T271; APC), anti-HLA-DR (Biolegend; clone L243; BV421) and analysed by flow cytometry (BD LSRII).

We first assessed published T-cell biomarkers [3, 4, 6-9] for their ability to distinguish LTBI participants from TB patients. Proportions of HLA-DR-expressing IFN $\gamma$-producing CD4 T-cells [3, 6, 7], CD27 median fluorescence index (MFI) ratio on IFN $\gamma$-producing CD4 T-cells [4] and frequencies of IL2-producing T-cells [8] following ESAT-6/CFP-10 stimulation were significantly lower in LTBI participants compared to TB patients, while proportions of single TNF-producing CD4 T-cells [9] were not different between the groups (figure 1a). Individuals with stimulated samples that were not statistically different (Fisher's exact test) from their unstimulated control were classified as non-responders and had uninterpretable phenotypic results. Only HLA-DR and the CD27 MFI ratio achieved area under the receiver operating characteristic (ROC) curves (AUC) $>0.8$ (figure 1a).

Next, we aimed to determine whether novel combinations of these published biomarkers could improve diagnostic performance. To achieve this a defined workflow was established (figure 1b). We only analysed

@ERSpublications

We compared candidate T-cell-based TB biomarkers and identified HLA-DR on Mtb-specific T-cells as a priority marker http://ow.ly/TyHa30iwVbL

Cite this article as: Musvosvi M, Duffy D, Filander E, et al. T-cell biomarkers for diagnosis of tuberculosis: candidate evaluation by a simple whole blood assay for clinical translation. Eur Respir J 2018; 51: 1800153 [https://doi.org/10.1183/13993003.00153-2018]. 

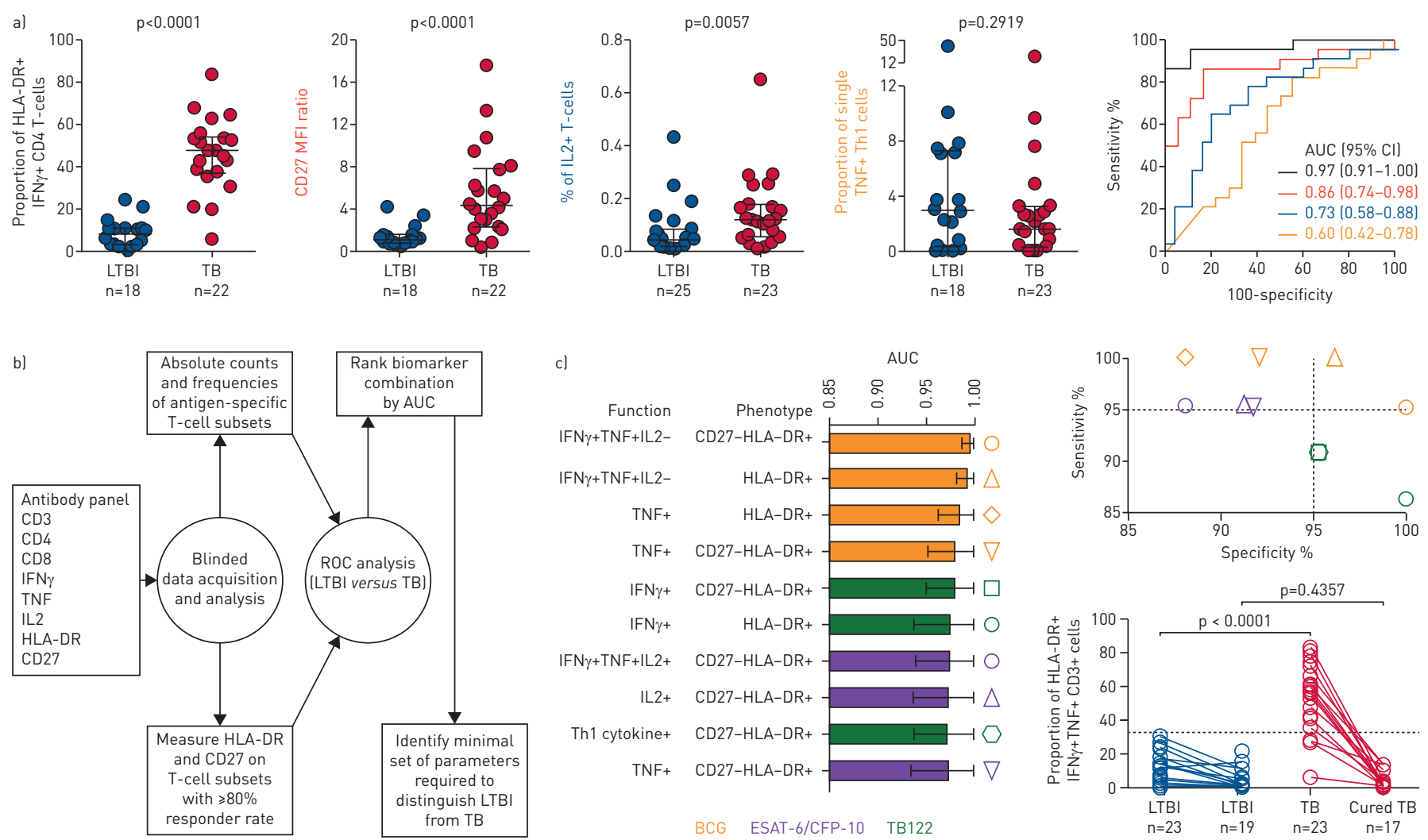

FIGURE 1 Assessment of candidate T-cell-based tuberculosis (TB) biomarkers and identification of a minimal set of parameters that distinguish persons with active TB from those with latent Mycobacterium tuberculosis infection (LTBI). a) Proportions of ESAT-6/CFP-10-specific CD4 T-cells, defined by IFN $\gamma$ production, expressing HLA-DR (black). CD27 median fluorescence index (MFI) ratios, calculated by dividing the CD27 MFI of total CD4 T-cells by the CD27 MFI on ESAT-6/CFP10-specific CD4 T-cells, defined by IFN $\gamma$ production (red). Frequencies of IL2-producing T-cells (CD4 and CD8) following ESAT-6/CFP-10 stimulation (blue). Proportions of ESAT-6/CFP-10-specific CD4T cells, defined by IFN $\gamma$, TNF, and/or IL2 production, producing TNF alone (orange). Phenotypic analysis was only performed on donors with a positive ESAT-6/CFP-10 response, defined using Fisher's exact test comparing unstimulated and stimulated samples. Comparison of the area under the receiver operating characteristic (ROC) curve $(A \cup C)$ of the published biomarkers. It is important to note that a larger CD27 MFI ratio indicates reduced CD27 expression by M. tuberculosis-specific CD4 T-cells. b) Analysis workflow used to assess the performance of novel biomarker combinations. Blinded analysis of FCS files was performed in FlowJo X. Tables of raw frequencies, counts, and MFI values were imported into R for analysis. Background subtraction (subtracting unstimulated frequencies from the stimulated frequencies), and absolute counts [13] of T-cell subsets were calculated in R. Prior to phenotypic analysis of specific T-cell subsets, the Fisher's exact test was used to determine if stimulation (i.e. BCG, ESAT-6/CFP-10, and TB122) induced a positive response (cytokine-positive counts significantly higher in stimulated than unstimulated sample). Responses were defined by $\mathrm{p}$ $<0.05$. c) Top 10 antigen-specific T-cell biomarker combinations ranked by the AUC (left plot). Only biomarker combinations detectable in more than $80 \%$ of patients and controls were evaluated. A total of 770 combinations were evaluated and the Benjamini Hochberg procedure was used to correct for multiple comparisons. All the top 10 biomarker combinations had a false discovery rate of $<0.001$. The pROC package in R was used to determine if AUCs were statistically different. Comparison of the sensitivity and specificity of each biomarker combination (top right plot). Summary graph on the bottom right depicts the proportions of HLA-DR+ ESAT-6/CFP-10-specific T-cells co-expressing IFN $\gamma$ and TNF in LTBI before and after follow up, and those with active TB before and after TB treatment. Dashed line indicates the cut-off threshold.

cytokine combinations with a responder rate exceeding $80 \%$ in each study group, to reduce bias and ensure biomarker robustness. HLA-DR expression appeared in all the top 10 ranked combinations and was therefore the most informative feature (figure 1c). It should be noted that only CD4 and no CD8 T-cell specific features made the top 10. While we observed a range of AUCs (and confidence intervals) in the selected combinations, these were not statistically different from each other, likely due to the relatively small sample size of our study cohort. We then utilised the Youden index to determine threshold cut-offs that maximised sensitivity and specificity for each biomarker. Only HLA-DR expression on BCG-specific IFN $\gamma+\mathrm{TNF}+\mathrm{IL2}-\mathrm{CD} 4 \mathrm{~T}$-cells (regardless of CD27) reached $95 \%$ specificity and $95 \%$ sensitivity (figure 1c).

To reduce the assay cost and simplify analysis, we then sought to reduce the number of fluorescent flow cytometry parameters, while maintaining a high level of diagnostic performance. Analysis of up to four fluorescent parameters can be performed on small and cheap flow cytometers, which are more accessible in resource-constrained settings. Based on the assay performance described above, we selected HLA-DR expression on IFN $\gamma$ and TNF co-expressing T-cells. Detection of antigen-specific T-cells co-expressing two cytokines was less affected by artefacts originating from autofluorescence or non-specific antibody staining, which are typically observed in single cytokine-producing cells (data not shown). In our dataset virtually 
all M. tuberculosis-specific T-cells were CD4+, but since others have proposed that measurement of CD8+ responses might yield additional diagnostic value [5], we reasoned that gating on total CD3+ cells would encompass responses from both $\mathrm{CD} 4+$ and $\mathrm{CD} 8+$ cells. Although the choice of antigenic stimulus did not appear to influence biomarker performance (figure 1c), we focused on stimulation with $M$. tuberculosis-specific antigens ESAT-6/CFP-10, which may provide the additional advantage of distinguishing between persons sensitised by $M$. tuberculosis infection from those not sensitised (i.e. M. tuberculosis uninfected). This would expand the applicability of this blood-based diagnostic test for TB disease to also include diagnosis of M. tuberculosis infection.

Based on these considerations, we reanalysed the flow cytometry data, focusing on HLA-DR expression by IFN $\gamma$ and TNF-expressing CD3+ T-cells using this four-colour panel, and ignored the presence of the other antibodies in the panel. LTBI participants were readily distinguishable from those with active TB (AUC 0.97, 95\%CI 0.91-1.00) (figure 1c). Importantly, expression of all the top 10 biomarkers (data not shown), and notably the proportion of HLA-DR+ M. tuberculosis-specific CD3+ T-cells co-expressing IFN $\gamma$ and TNF (assessed with the four-colour panel; figure 1c), dropped to levels similar to those observed in healthy controls following successful treatment of TB patients.

Our study independently validated published T-cell diagnostic biomarkers using a whole blood-based approach, which is more amenable to translation to field testing than peripheral blood mononuclear cells (PBMCs). We systematically assessed all possible combinations of previously validated biomarkers and compared them head-to-head in the same population. Our results are in agreement with recent work showing that better discrimination was observed with HLA-DR expression compared to CD27 in PBMCs [7]. While previous studies have focused on measuring expression of HLA-DR or CD27 by M. tuberculosis-specific T-cells identified solely by IFN $\gamma$ expression $[3,5]$, our results suggest that a combination of two functional outcomes with phenotypic profiles may yield better diagnostic accuracy. Importantly, we demonstrate potential feasibility for a test based on a limited flow cytometry panel consisting of only four antibodies, which yielded a sensitivity of $86 \%$ and a specificity of $100 \%$ for diagnosis of active TB disease. These performance characteristics fall within the minimum target product profile recommended by a World Health Organization consensus meeting [14]. Successful treatment of TB resulted in these biomarkers returning to levels observed in healthy persons with asymptomatic $M$. tuberculosis infection, corroborating high specificity for TB.

Although our study did not include participants co-infected with HIV, recent data demonstrated that HLA-DR expression on $M$. tuberculosis-specific T-cells was significantly higher in HIV-infected persons with active TB as compared to latently infected controls $[6,7]$. These results suggest that the simplified whole blood TB diagnostic assay likely has applicability in persons living with HIV. Key future steps are to determine the performance of T-cell-based biomarkers in HIV-infected and paediatric populations, including control groups of patients with other respiratory diseases. In conclusion, our data support further development of a simplified whole blood assay to measure HLA-DR expression on $M$. tuberculosis-specific T-cells for TB diagnosis and/or treatment monitoring.

Munyaradzi Musvosvi ${ }^{1}$, Darragh Duffy ${ }^{2,3}$, Elizabeth Filander ${ }^{1}$, Hadn Africa $^{1}$, Simbarashe Mabwe ${ }^{1}$, Lungisa Jaxa ${ }^{1}$, Nicole Bilek ${ }^{1}$, Alba Llibre ${ }^{2,3}$, Vincent Rouilly ${ }^{2,3}$, Mark Hatherill ${ }^{1}$, Matthew Albert ${ }^{2,3,4}$, Thomas J. Scriba ${ }^{1,5}$ and Elisa Nemes $\oplus^{1,5}$

${ }^{1}$ South African Tuberculosis Vaccine Initiative (SATVI), Institute of Infectious Disease and Molecular Medicine, and Division of Immunology, Dept of Pathology, University of Cape Town, Cape Town, South Africa. ${ }^{2}$ Laboratory of Dendritic Cell Immunobiology, Dept of Immunology, Institut Pasteur, Paris, France. ${ }^{3}$ INSERM U1223, Paris, France. ${ }^{4}$ Cancer Immunology, Genentech, Inc., South San Francisco, CA, USA. ${ }^{5}$ These authors contributed equally.

Correspondence: Munyaradzi Musvosvi, University of Cape Town, South African Tuberculosis Vaccine Initiative, Wernher and Beit South Building, Room S 2.01, Health Sciences Faculty, Cape Town, Western Cape, South Africa. E-mail: Munyaradzi.Musvosvi@uct.ac.za

Received: Dec 022017 | Accepted after revision: Feb 122018

Support statement: This work was supported by the Bill and Melinda Gates Foundation (OPP1114368), the Laboratoire d'Excellence "Milieu Intérieur" (ANR-10-LABX-69-01), and the European Commission-funded TBVAC2020 consortium (H2020-PHC-643381). M. Musvosvi was supported by the Carnegie Corporation of New York. Elisa Nemes is a Marylou Ingram Scholar. Funding information for this article has been deposited with the Crossref Funder Registry.

Conflict of interest: T.J. Scriba reports grants received from BMGF by University of Cape Town, during the conduct of the study. E. Nemes received grants from the Bill and Melinda Gates Foundation, during the conduct of the study.

\section{References}

1 World Health Organization. WHO Global tuberculosis report 2017. Geneva, World Health Organization, 2017. Available from: www.who.int/tb/publications/global_report/en/

$2 \quad$ Pai M, Behr MA, Dowdy D, et al. Tuberculosis. Nat Rev Dis Prim 2016; 2: 16076. 
3 Adekambi T, Ibegbu CC, Cagle S, et al. Biomarkers on patient $\mathrm{T}$ cells diagnose active tuberculosis and monitor treatment response. J Clin Invest 2015; 125: 1827-1838.

4 Portevin D, Moukambi F, Clowes P, et al. Assessment of the novel T-cell activation marker-tuberculosis assay for diagnosis of active tuberculosis in children: a prospective proof-of-concept study. Lancet Infect Dis 2014; 14: 931-938.

5 Rozot V, Patrizia A, Vigano S, et al. Combined use of Mycobacterium tuberculosis-specific CD4 and CD8 T-cell responses is a powerful diagnostic tool of active tuberculosis. Clin Infect Dis 2015; 60: 432-437.

6 Wilkinson KA, Oni T, Gideon HP, et al. Activation profile of Mycobacterium tuberculosis-specific CD4+ T cells reflects disease activity irrespective of HIV status. Am J Respir Crit Care Med 2016; 193: 1307-1310.

7 Riou C, Berkowitz N, Goliath R, et al. Analysis of the phenotype of Mycobacterium tuberculosis-specific CD4+ T cells to discriminate latent from active tuberculosis in HIV-Uninfected and HIV-Infected individuals. Front Immunol 2017; 8 .

8 Sargentini V, Mariotti S, Carrara S, et al. Cytometric detection of antigen-specific IFN- $\gamma /$ IL-2 secreting cells in the diagnosis of tuberculosis. BMC Infect Dis 2009; 9: 99.

9 Harari A, Rozot V, Enders FB, et al. Dominant TNF- $\alpha+$ Mycobacterium tuberculosis-specific CD4+ T cell responses discriminate between latent infection and active disease. Nat Med 2011; 17: 372-376.

10 Goletti D, Petruccioli E, Joosten SA, et al. Tuberculosis biomarkers: from diagnosis to protection. Infect Dis Rep 2016; 8: 24-32.

11 Lindestam Arlehamn CS, McKinney DM, Carpenter C, et al. A quantitative analysis of complexity of human pathogen-specific CD4T cell responses in healthy M. tuberculosis infected South Africans. PLoS Pathog 2016; 12: $1-27$.

12 Kagina BM, Mansoor N, Kpamegan EP, et al. Qualification of a whole blood intracellular cytokine staining assay to measure mycobacteria-specific CD4 and CD8T cell immunity by flow cytometry. J Immunol Methods 2015; 417: 22-33.

13 Nemes E, Kagina BMN, Smit E, et al. Differential leukocyte counting and immunophenotyping in cryopreserved ex vivo whole blood. Cytom Part A 2015; 87: 157-165.

14 Denkinger CM, Kik S V, Cirillo DM, et al. Defining the needs for next generation assays for tuberculosis. J Infect Dis 2015; 211: S29-S38. 